\title{
LEPTOSPIROSE NO ESTADO DO PARANÄ, BRASIL: UMA ABORDAGEM DE SAÚDE ÚNICA
}

\section{Leptospirosis in the state of Paraná, Brazil: A One Health Approach}

Igor Massahiro de Souza Suguiura ${ }^{1}$

1. Mestre em Patologia Experimental pela Universidade Estadual de Londrina. Médico Veterinário da Divisão de Vigilância em Saúde, 17a Regional de Saúde/Londrina-PR. ORCID: https://orcid.org/00000001-7160-319X.

CONTATO: Igor Massahiro de Souza Suguiura | Endereço: Alameda Manoel Ribas n01 | Londrina-PR | Telefone: (43)3379-6122 | E-mail: Igor.suguiura@sesa.pr.gov.br

COMO CITAR: Suguiura IMS. Leptospirose no estado do Paraná, Brasil: uma abordagem de saúde única. R. Saúde Públ. Paraná. 2019 Dez;2(2):77-84.

\footnotetext{
(c) (i) COPYRIGHT Esta obra é disponibilizada nos termos da Licença Creative Commons - 4. 0
} Internacional. É permitida a reprodução parcial ou total desta obra, desde que citada a fonte.

\begin{abstract}
RESUMO Leptospirose é uma doença endêmica no Brasil. Sua ocorrência é relacionada a diversos fatores de vulnerabilidade social e exposição ocupacional, sendo sua cadeia epidemiológica complexa, envolvendo o homem, animais e meio ambiente. Foi realizado um estudo transversal descritivo dos casos novos de leptospirose confirmados no estado do Paraná entre os anos de 2007-2017. A incidência média para o período foi de 2,98 casos por 100.000 habitantes com letalidade média de 10,53 óbitos por 100 casos. A maior parcela da população afetada foi do sexo masculino $(84,4 \%)$, cor branca $(72,9 \%)$ habitantes do perímetro urbano $(56,2 \%)$ que se infectaram no domicilio $(40,4 \%)$, sendo em sua maioria confirmados por análises laboratoriais (93,3\%). A doença apresentou aumento em comparação à sua incidência no período de 1993 a 2001 no estado do Paraná, com distribuição sazonal ao longo do ano
\end{abstract}

PALAVRAS-CHAVE: Leptospirose. Epidemiologia. Saúde Única. 
ABSTRACT Leptospirosis is an endemic disease in Brazil. Its occurrence is related to several factors of social vulnerability and occupational exposure, with its complex epidemiological chain involving man, animals, and environment. A descriptive cross-sectional study of new confirmed leptospirosis cases in the State of Paraná between 2007-2017 was performed. The average incidence for the period was 2.98 cases per 100,000 inhabitants with an average lethality of 10.53 deaths per 100 cases. The largest portion of the affected population was male (84.4\%), white (72.9\%) living in the urban perimeter (56.2\%) and infected at home (40.4\%). In addition, most of the cases were confirmed by laboratory analysis (93.3\%). The disease showed an increase when compared to its incidence in the period from 1993 to 2001 in the state of Paraná, with seasonal distribution throughout the year.

KEYWORDS: Leptospirosis. Epidemiology. One Health.

\section{INTRODUÇÃO}

A leptospirose é uma zoonose distribuída mundialmente, com ocorrência comum em paises pobres e em desenvolvimento1. A doença pode ter manifestação clínica ou subclínica causada pela espiroqueta pertencente ao gênero Leptospira, família Leptospiraceae, que possui taxonomia molecular complexa e não padronizada². Atualmente são descritas 35 espécies pertencentes ao gênero Leptospira3. Em contrapartida, a sua classificação sorológica é amplamente utilizada na rotina clínica, sendo identificados mais de 300 sorovares diferentes ${ }^{2}$.

A sua capacidade de infectar e se alojar nos túbulos renais, sendo eliminada pela urina, de diversas espécies animais torna esta bactéria um importante microorganismo zoonótico ${ }^{4-5}$. Esta pode permanecer por longos períodos no meio ambiente, quando as condições são favoráveis, ${ }^{4}$ sendo que a infecção ocorre através da penetração de mucosas, pele lesionada ou mesmo íntegra quando exposta a longos períodos de contato com a água ${ }^{6-7}$.

O tipo de teste laboratorial preconizado para o diagnóstico varia conforme a fase da doença. A maioria dos laboratórios oficiais do Brasil utiliza o ELISA-IgM e o teste de aglutinação microscópica (MAT) ${ }^{7}$, sendo o MAT considerado o padrão ouro para a identificação dos sorovares que ocorrem na população animal e humana1,7,8.

Uma abordagem de saúde única é de grande valia para o estudo de doenças que, como a leptospirose, possuem uma cadeia epidemiológica complexa envolvendo homem, animal e meio 
ambiente. A manifestação clínica da leptospirose pode se confundir com outras doenças icterohemorrágicas. Por isso é preciso atentar-se às subnotificações da doença, principalmente em regiões com prevalência de outros agravos com manifestação clínica semelhante ${ }^{9}$. O presente trabalho descreveu o perfil epidemiológico e a distribuição espaço-temporal da leptospirose no estado do Paraná, Brasil, no período de 2007 a 2017, tendo em vista subsidiar discussões sobre estratégias de vigilância e promoção de saúde com abordagem em saúde única.

\section{METODOLOGIA}

Foi realizado um estudo transversal descritivo dos casos notificados e confirmados de leptospirose no estado do Paraná, nos anos de 2007 a 2017. O estado do Paraná é localizado no Sul do Brasil fazendo fronteira com os estados de São Paulo, Santa Catarina e com os países Argentina e Paraguai. Possui comunicação com o Oceano Atlântico em um território de 199.880.200 km² dividido em 399 municípios.

A leptospirose é uma doença icterohemorrágica de notificação compulsória nacional10 ${ }^{10}$ Os antecedentes epidemiológicos foram obtidos a partir do Banco de Informação de Agravos de Notificação - SINAN do Ministério da Saúde/SVS disponível de forma livre em "Informações de saúde" (DATASUS, TABNET; Epidemiológicas e morbidade). Os dados estimados da população do Paraná para o período deste estudo foram obtidos no website do DATASUS (http://www2.datasus.saude.gov.br), que utilizou a população total estimada a partir do Instituto Brasileiro de Geografia e Estatística (IBGE).

Para a caracterização epidemiológica foram utilizadas as seguintes variáveis: Sexo, cor, faixa etária, área de infecção, local de infecção, evolução, critério de confirmação, casos novos confirmados a partir do ano de primeiros sintomas e casos confirmados para o mês de primeiros sintomas. A análise epidemiológica baseou-se na distribuição temporal dos casos de residentes do estado do Paraná entre os anos de 2007 e 2017, assim como os indicadores de incidência e letalidade.

A análise descritiva foi expressa como frequência, média e/ou porcentagem, com análise estatística e confecção dos gráficos realizadas utilizando o programa Microsoft Office Excel $₫$ 2010.

\section{RESULTADOS}

Foram confirmados e notificados no intervalo de 2007 a 2017, 3.559 casos de leptospirose no estado do Paraná, correspondendo a 25,8\% dos casos notificados na região sul do Brasil (3.559/13.816). No estado do Paraná, o maior volume de notificações corresponde aos meses de janeiro a março e o menor volume entre julho a setembro (Figura 1).

As incidências variaram de 1,87 a 4,57 casos por 100 mil habitantes (incidência média de 2,98; \pm $1 \mathrm{DP})$, sendo que a letalidade variou de 4,38 a 18,5 óbitos por 100 casos (letalidade média 10,53 $\pm 3,68$ DP) (Figura 2).

Em relação às características sociodemográficas dos casos confirmados (Tabela 1), os grupos majoritários de indivíduos afetados são: por sexo, masculino (84,4\%, em proporção de 5:1); por idade, na faixa de 20-39 anos (35,9\%); por raça ou cor, branca $(72,9 \%)$ e, por local de residência, habitantes da área urbana (56,2\%). Em relação ao local de infecção mais provável, o domicílio foi o predominante $(40,4 \%)$.

Do total de casos confirmados a maioria dos pacientes (93,3\%) teve seu diagnóstico definido por critério laboratorial, seguido por clínico epidemiológico (5.7\%), sendo observado $1 \%$ de campos ignorados ou brancos. 
Figura 1 Casos confirmados e notificados por mês de primeiros sintomas no estado do Paraná no período de 2007 a 2017.

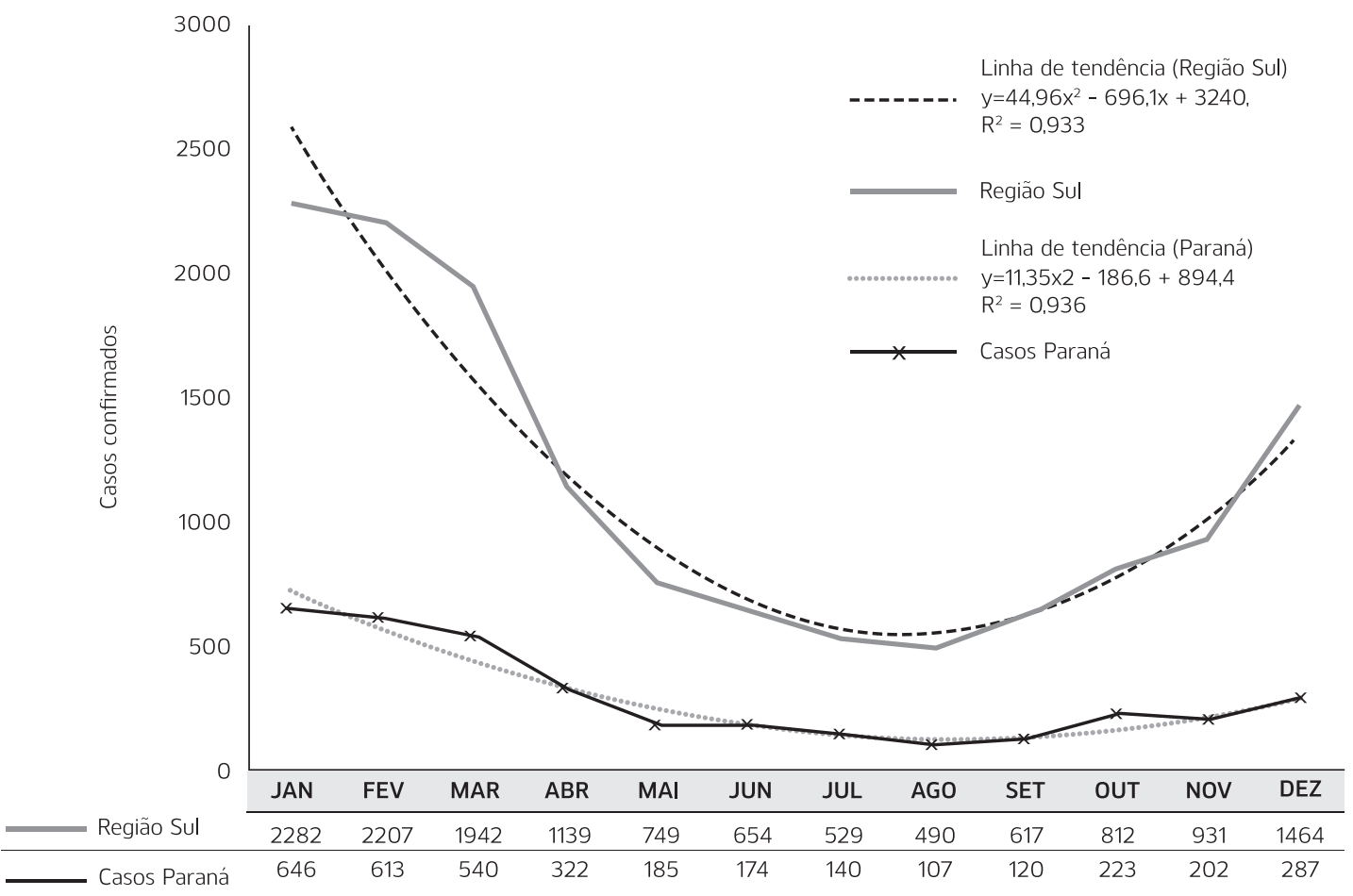

Fonte: Elaborado pelos autores (2019)

Figura 2 Coeficiente de incidência e taxa de letalidade de leptospirose no estado do Paraná, 2007 a 2017

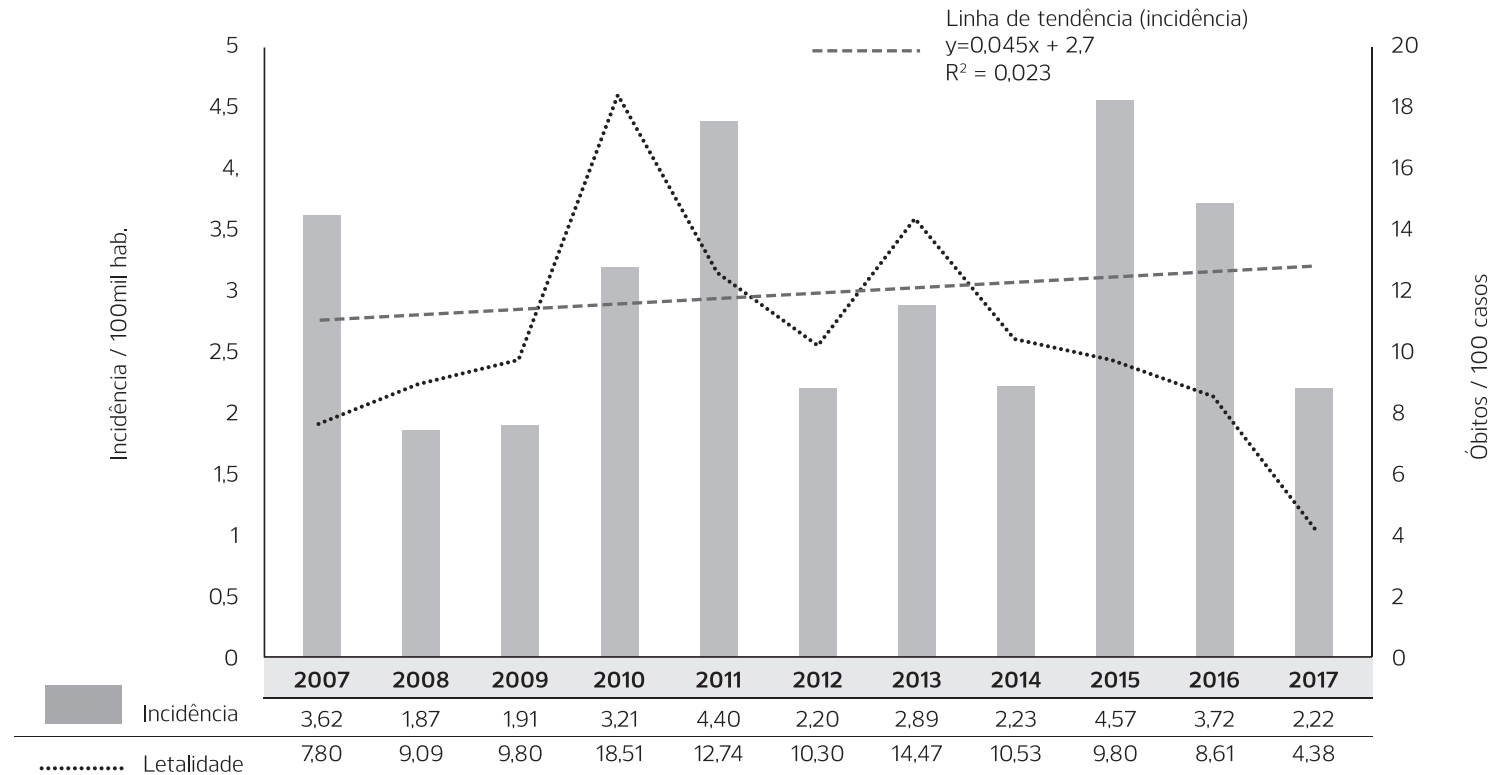

Fonte: Elaborado pelos autores (2019) 
Tabela 1 Características sociodemográficas dos casos confirmados de leptospirose no estado do Paraná entre os anos de 2007-2017.

\begin{tabular}{|c|c|c|}
\hline CATEGORIA & $\mathbf{n}$ & $\%$ \\
\hline \multicolumn{3}{|l|}{ Sexo } \\
\hline Maculino & 2.994 & 84,4 \\
\hline Feminino & 555 & 15,6 \\
\hline \multicolumn{3}{|l|}{ Raça/Cor } \\
\hline Branca & 2.586 & 72,9 \\
\hline Parda & 548 & 15,4 \\
\hline Preta & 116 & 3,3 \\
\hline Amarela & 25 & 0,7 \\
\hline Indígena & 7 & 0,2 \\
\hline Ignorado & 267 & 7,5 \\
\hline \multicolumn{3}{|l|}{ Faixa etária } \\
\hline$<1-4$ & 26 & 0.7 \\
\hline $5-9$ & 70 & 1,9 \\
\hline $10-14$ & 258 & 7,1 \\
\hline $15-19$ & 382 & 10,6 \\
\hline $20-39$ & 1.295 & 35,9 \\
\hline $40-59$ & 1.197 & 33,2 \\
\hline $60-64$ & 137 & 3,8 \\
\hline $65-69$ & 101 & 2,8 \\
\hline $70+$ & 89 & 2,5 \\
\hline Ignorado/Branco & 54 & 1,5 \\
\hline \multicolumn{3}{|c|}{ Local de infecção área } \\
\hline Urbana & 1.996 & 56,2 \\
\hline Rural & 950 & 26,7 \\
\hline Periurbana & 132 & 3,7 \\
\hline Ignorado & 475 & 13,4 \\
\hline \multicolumn{3}{|c|}{ Local da infecção ambiente } \\
\hline Domiciliar & 1.435 & 40,4 \\
\hline Trabalho & 722 & 20,3 \\
\hline Lazer & 506 & 14,3 \\
\hline Outro & 169 & 4,8 \\
\hline Ignorado/Branco & 717 & 20,2 \\
\hline
\end{tabular}

Fonte: Elaborado pelos autores (2019)

\section{DISCUSSÃO}

A leptospirose apresenta características de endemicidade no estado do Paraná11. Os coeficientes de incidência apresentaram tendência de aumento. Partindo da série histórica de 1993 a $2001^{11}$. Houve aumento da incidência $(2,01 ; \pm 0,86$ DP) para a série de 2007-2017 (2,98; \pm 1 DP). Uma provável explicação para o aumento da incidência seria o aumento da sensibilidade das equipes de saúde na detecção dos casos suspeitos.

A leptospirose é sabidamente uma doença vinculada à veiculação hídrica tanto por atividades aquáticas quanto por deficiência em saneamento básico1. A doença no Paraná apresenta uma distribuição sazonal ao longo do ano (Figura 1), sendo observada a correlação entre os meses com maiores índices pluviométricos e os meses de maior número de notificações, como descrito por Paula12. Tal comportamento é também observado em outras regiões do Brasil, nas quais o aumento de notificações acompanha a curva de pluviosidade da região observada ${ }^{12-15}$.

Alguns estudos verificaram a presença de água como fator de risco para a doença também em animais. Em estudo realizado em granja de suínos, Delbem e colaboradores ${ }^{16}$ verificaram que a presença de bebedouros do tipo canaleta, assim como a presença de áreas alagadiças próximas às instalações dos animais e a falta de limpeza dos reservatórios de água foram consideradas fatores de risco para a soropositividade à doença, sendo que neste estudo o sorovar icterohaemorrhagiae foi o mais frequente, sendo o rato seu principal reservatório ${ }^{17}$.

O caráter hídrico da veiculação da doença é comumente observado nos casos de surtos em humanos após enchentes, mas também se relaciona à deficiência de saneamento básico, como observado em estudos de áreas de baixa renda de Curitiba que utilizaram o cão como animal sentinela18-20. O maior número de casos em ambiente urbano no Paraná é consoante aos resultados obtidos por Arsky et al. ${ }^{21}$ a partir de dados epidemiológicos nacionais. Sendo a leptospirose em área urbana relacionada à falta de acesso a saneamento básico, baixos níveis socioeconômicos e precariedade de domicílio e peridomicílio ${ }^{22}$. 
Além da presença de roedores como fator de risco para a doença, ${ }^{23-24}$ a participação de outros animais domésticos em meio urbano na cadeia epidemiológica da leptospirose não pode ser esquecida, pois atuam como portadores e potenciais transmissores. Pela proximidade com os humanos, os cães podem ser importantes carreadores da doença no meio urbano ${ }^{4}$, sendo estes os reservatórios naturais do sorovar canicola ${ }^{25}$. Estudos conduzidos no estado do Paraná detectaram cães sorologicamente positivos em Curitiba ${ }^{18-20}$, Bandeirantes ${ }^{26}$, Umuarama ${ }^{27-28}$, Jaguapitã29, Jataizinho ${ }^{30-31}$, Assis Chateaubriand, Marechal Cândido Rondon, Moreira Sales, Paranavaí, Pérola, São Jorge do Patrocínio ${ }^{28}$. Rio Branco do Ivaí, Rosário do Ivaí, Telêmaco Borba, Leópolis, Nova Fátima, Congoinhas, Assaí, Rolândia ${ }^{32}$ e em Londrina ${ }^{33-35}$, sendo que neste último município foram detectados cães errantes com bacteriúria ${ }^{34}$. Em revisão sistemática de 19 trabalhos em diferentes paises que avaliaram o impacto dos cães na ocorrência da leptospirose em humanos foi indicado que a presença destes animais não estaria associada com a doença em alguns contextos ${ }^{24}$. No entanto foram identificados casos em que os cães atuariam como pontes entre os reservatórios silvestres e o homem ${ }^{36}$, ou mesmo contaminando a água de enchentes com as bactérias eliminadas pela urina ${ }^{37}$.

A leptospirose foi primeiramente reconhecida como uma doença ocupacional relacionada a atividades agrícolas, manejo de animais e manutenção de redes de esgoto 4 . A proximidade de animais e humanos torna os trabalhadores rurais, médicos veterinários e magarefes uma população de risco para a infecção pela bactéria ${ }^{10}$. Inquéritos sorológicos em rebanhos bovinos, assim como a detecção de animais positivos em abatedouros no Paraná mostram ampla distribuição de animais soropositivos e o potencial risco aos trabalhadores que têm contato com os animais. A leptospirose é considerada uma das maiores causas infecciosas para a ocorrência de problemas reprodutivos em bovinos e outros ruminantes ${ }^{38}$. As ocorrências de aborto, natimortalidade, infertilidade, entre outros problemas reprodutivos causados pela doença tornam o manejo desses animais um risco para os trabalhadores do setor pecuário. O perfil ocupacional da doença foi explorado em inquéritos sorológicos por alguns autores no Paraná como Garcia e Navarro ${ }^{39}$, que observaram o auxílio ao parto em animais como um possivel fator de risco no município de Guaraci. Ramos Vieira et al. ${ }^{40}$, em 1993, obteve positividade de 25.9\% em trabalhadores rurais de Londrina, enquanto Gonçalves et al. ${ }^{41}$ obteve uma positividade de $12,1 \%$ em Jaitizinho-PR, sendo que os fatores de risco para adquirir a infecção incluiam auxílio ao parto, castração, abate, e outras formas de contato com animais. Gonçalves et al. ${ }^{41}$ realizou testes sorológicos a partir de amostras obtidas de trabalhadores em um abatedouro de bovinos no norte do Paraná obtendo uma positividade de 4\% (2006), enquanto Ramos Vieira et al. $^{40}$, em estudo semelhante, obteve positividade de mais de $24 \%$ em açougueiros do município de Londrina. Apesar da correlação entre o estreito contato com os animais e a leptospirose. deve-se salientar o aspecto ambiental da doença, pois tais trabalhadores, além da exposição durante o manejo dos bovinos, também estão expostos ao contato com outros prováveis carreadores da doença, água ou solo contaminado.

A presença e disseminação ambiental de espiroquetas viáveis também apresentam risco a outras categorias ocupacionais que não estão relacionadas ao contato direto com animais. Vasconcelos et. $\mathrm{al}^{42}$, em estudo com profissionais de diferentes categorias em Londrina, encontrou positividade de 35\% em funcionários de limpeza pública, em comparação aos 5\% observados em um público sem contato com animais ou material suspeito de contaminação. No entanto, apesar de a infecção no local de trabalho ser a segunda colocada em ordem de frequência no estado do Paraná, é quase superada pelo total de campos brancos e ignorados para local de infecção. A falta de completude das fichas de notificação dificulta a análise epidemiológica dos agravos de forma geral. 
Ações de vigilância e promoção em saúde são essenciais para o controle da incidência da doença. Projetos de gestão municipal visando à prevenção de novos casos, assim como à implementação de saneamento básico e atenção a populações vulneráveis são essenciais no controle da doença, dado estarem intimamente ligados aos seus fatores de risco ${ }^{1,7}$. Medidas emergenciais nos períodos de chuva intensa e enchentes como a quimioprofilaxia ${ }^{43}$ e realocação de grupos expostos, são medidas a serem realizadas para minimizar a transmissão da leptospirose por via hídrica em tais situações. Em relação à saúde do trabalhador, a conscientização dos trabalhadores expostos à infecção quanto ao uso de equipamentos de proteção individual é essencial'17. A identificação e o adequado tratamento dos animais acometidos é uma importante forma de reduzir o risco de infecção humana e animal. A implementação de um sistema de notificação em saúde pública voltado a ocorrência de doenças zoonóticas em animais seria de grande ajuda na investigação e prevenção de casos humanos de leptospirose.

\section{CONSIDERAÇÕES FINAIS}

Uma grande limitação aos estudos que utilizam dados oriundos de fichas de notificação é a incompletude das informações obtidas. Em relação à leptospirose no Estado do Paraná, não é diferente. A apropriação da importância das notificações de agravos deve ser intrínseca aos profissionais da saúde, pois são importantes ferramentas para a promoção desta. Ao analisar os casos confirmados de leptospirose no estado do Paraná, conseguimos visualizar um aumento da incidência da doença ao longo da série histórica, sendo necessário o aperfeiçoamento das ações para controle, sobretudo a adoção de ações sob o conceito de saúde única, tendo em vista a estreita relação entre homem, animal e meio ambiente nesta enfermidade.

\section{REFERÊNCIAS}

1. World Health Organization. Human leptospirosis : guidance for diagnosis, surveillance and control [Internet]. Geneva: World Health Organization; 2003 [citado em 2019 Ago 20]. Disponivel em: http://www.who.int/iris/handle/10665/42667.

2. Guglielmini J, Bourhy P. Schiettekatte O, Zinini F. Brisse S Picardeau M. Genus-wide Leptospira core genome multilocus sequence typing for strain taxonomy and global surveillance. PLoS Negl Trop Dis [Internet]. 2019 [citado em 2019 Out 10]:13(4) :e0007374. Disponivel em: https://journals.plos.org/plosntds/ article?id=10.1371/journal.pntd.0007374.

3. Thibeaux R, Iraola G, Ferrés I, Bierque E, Girault D, Soupé-Gilbert $\mathrm{M}-\mathrm{E}$, et al. Deciphering the unexplored Leptospira diversity from soils uncovers genomic evolution to virulence. Microb Genom. 2018:4(1)

4. Guerra MA. Leptospirosis. J Am Vet Med Assoc 2009:234(4):472-8

5. Schuller S, Francey T, Hartmann K, Hugonnard M, Kohn B, Nally $J E_{\text {, et }}$ al. European consensus statement on leptospirosis in dogs and cats. J Small Anim Pract. 2015:56(3):159-79.

6. Adler B, Moctezuma ADLP. Leptospira and leptospirosis. Vet Microbiol. 2010:140(3-4):287-96.

7. Brasil, Ministério da Saúde. Leptospirose e manejo clínico [Internet]. Brasília: Ministério da Saúde; 2014 [citado em 2019 Ago 21]. Disponível em: http://portalarquivos.saude.gov.br/images/ pdf/2014/dezembro/02/Miolo-manual-Leptospirose17-9-2014. pdf

8. World Organisation for Animal Health. Manual of diagnostic test and vaccines for terrestrial animals [Internet]. Paris: OIE: 2018 [citado 2019 Ago 21]. Disponivel em: https://www.oie.int/ fileadmin/Home/eng/Health_standards/tahm/3.01.12_LEPTO.pdf

9. Bharti AR, Nally JE, Ricaldi JN, Matthias MA, Diaz MM, Lovett MA, et al. Leptospirosis: a zoonotic disease of global importance. Lancet Infect Dis. 2003:3(12):757-71.

10. Brasil, Ministério da Saúde, Secretaria de Vigilância em Saúde. Guia de vigilância em saúde. Brasília: Ministério da Saúde: 2017.

11. Paraná, Secretaria de Estado da Saúde. Leptospirose dados Paraná e informações [Internet]. Curitiba: SESA; 2002 [citado 2019 Set 1]. Disponivel em: http://www.saude.pr.gov.br/modules/ conteudo/conteudo.php?conteudo=479.

12. Paula EV. Leptospirose Humana: uma análise climatogeográfica de sua manifestação no Brasil, Paraná e Curitiba. In Anais XII Simpósio Brasileiro de Sensoriamento Remoto, 2005 Goiânia. São José dos Pinhais: INPE: 2005. p. 16-21.

13. Guimarães RM, Cruz OG, Parreira VG, Mazoto ML, Vieira JD, Asmus CIRF. Análise temporal da relação entre leptospirose e ocorrência de inundações por chuvas no município do Rio de Janeiro, Brasil, 2007-2012. Ciên Saúde Colet. 2014;19(9):3683-92.

14. Lara JM, Zuben AV, Costa JV, Donalisio MR, Francisco PMSB. Leptospirose no município de Campinas, São Paulo, Brasil: 2007 a 2014. Rev Bras Epidemiol. 2019:22:1-13.

15. Lima RJDS, Abreu EMND, Ramos FLDP, Santos RDD, Santos DDD, Santos FAAD, et al. Análise da distribuição espaço-temporal 
da leptospirose humana em Belém, Estado do Pará, Brasil. Rev Pan Amaz Saúde. 2012:3(2):33-40.

16. Delbem ÁCB, Freire RL, Silva CAD, Müller EE, Dias RA, Neto JSF, et al. Fatores de risco associados à soropositividade para leptospirose em matrizes suínas. Ciênc Rural. 2004;34(3):847-52.

17. Bharti AR, Nally JE, Ricaldi JN, Matthias MA, Diaz MM, Lovett $M A$, et al. Leptospirosis: a zoonotic disease of global importance. Lancet Infect Dis. 2003:3(12):757-71.

18. Tesserolli GL, Alberti JVA, Agottani JVB, Fayzano L, Warth JFG. Soroprevalência para leptospirose em cães de Curitiba, Paraná Rev Acad Ciênc Animal. 2005:3(4):35-8.

19. Martins CM. Barros CC, Galindo CM. Kikuti M. Ullmann LS. Pampuch RDS, et al. Incidence of canine leptospirosis in the metropolitan area of Curitiba, State of Parana, Southern Brazil. Rev Soc Bras Med Trop. 2013:46(6):772-5

20. Bier D, Shimakura SE, Morikawa VM, Ullmann LS, Kikuti M, Langoni H. et al. Análise espacial do risco de leptospirose canina na Vila Pantanal. Curitiba, Paraná. Pesqui Vet Bras. 2013:33(1):74-9.

21. Arsky MLS, Oliveira WK, Oliveira RC, Arruda AS. Probable areas of infection and ambience of occurrence of human leptospirosis in Brazil (2001- 2003). Rev Cubana Med Trop. 2005; 57(1):59-60.

22. Pelissari DM, Maia-Elkhoury ANS, Arsky MLNS, Nunes ML. Revisão sistemática dos fatores associados à leptospirose no Brasil, 2000-2009. Epidemiol Serv Saúde. 2011:20(4):565-74.

23. Sarkar U, Ko Al, Kalofonos I, Flannery B, Grunstein I, Reis MG, et al. Population-based case-control investigation of risk factors for leptospirosis during an urban epidemic. Am J Trop Med Hyg. 2002:66(5):605-10.

24. Mwachui MA, Crump L, Hartskeerl R, Zinsstag J, Hattendorf J. Environmental and behavioural determinants of leptospirosis transmission: A systematic review. PLoS Negl Trop Dis. 2015:9(9).

25. André-Fontaine G. Canine leptospirosis - Do we have a problem? Vet Microbiol. 2006:117(1):19-24

26. Zacarias FGDS, Marques DRC, Cardoso MJL, Freitas JCD, Junior AZ, Zamarian TP. Frequência de anticorpos anti-Leptospira spp. em cães atendidos no hospital veterinário da universidade estadual do norte do Paraná - UNEP. Arq Ciênc Vet Zool UNIPAR. 2015:17(2)91-5

27. Dreer MKDP, Gonçalves DD, Caetano ICDS, Gerônimo E, Menegas PH. Bergo D, et al. Toxoplasmosis, leptospirosis and brucellosis in stray dogs housed at the shelter in Umuarama municipality, Paraná, Brazil. J Venom Anim Toxins Incl Trop Dis. 2013;19(1):23-7.

28. Hafemann DCM, Merlini LS, Gonçalves DD, Fortes MS, Navarro IT. Chiderolli RT, et al. Detection of anti-Leptospira spp., antiBrucella spp., and anti-Toxoplasma gondii antibodies in stray dogs. Semina Ciênc Agrar. 2018;39(1):167-76.

29. Hashimoto VY, Garcia JL, Spohr KAH, Silva FGD, Alves LA, Freitas JD. Prevalência de anticorpos contra Leptospira spp. em bovinos, caninos, equinos, ovinos e suínos do município de Jaguapitã, estado do Paraná, Brasil. Arq Inst Biol.2010:77(3):521-4.

30. Gonçalves DD, Benitez A, Lopes-Mori FMR, Alves LA, Freire RL, Navarro IT, et al. Zoonoses in humans from small rural properties in Jataizinho, Parana, Brazil. Braz J Microbiol. 2013:44(1):125-31.
31. Benitez ADN, Gonçalves DD. Freire RL, Rodrigues WB, Souza VRAD, Barbara JCA, et al. Seroepidemiology of leptospirosis in pet dogs in the urban area of the municipality of Jataizinho, Paraná. Semina Ciênc Agrar. 2012:33(Supl2):3201-10

32. Belitardo DR, Freitas JO, Müller EE. Leptospirose em animais do biotério central do Centro de Ciências Biológicas da Universidade Estadual de Londrina. Semina Ciênc Agrar. 2000;21(1):19-25.

33. Querino AMV, Delbem ACB, Oliveira RC, Silva FGD, Müller EE, Freire $\mathrm{RL}$, et al. Fatores de risco associados à leptospirose em cães do municipio de Londrina-PR. Semina Ciênc Agrar. 2003;24(1):2733

34. Benitez AN, Rodrigues GG, Gonçalves DD, Burke JC, Alves LA. Müller EE, et al. Leptospirose em cães errantes encontrados em campus universitário: avaliação sorológica e exame direto da urina. Semina Ciênc Agrar. 2010:31(1):191-6.

35. Caldart ET, Constantino C, Pasquali AKS, Benitez ADN, Hamada FN, Dias RCF, et al. Zoonosis in dogs and cats attended by the Birth Control Project: Toxoplasma gondii, Leishmania spp. and Leptospira spp., serodiagnosis and epidemiology. Semina Ciênc Agrar. 2015;36(1):253-65.

36. Céspedes $M$, Ormaeche $M$, Condori $P$, Balda L, Glenny M. Prevalencia de leptospirosis y factores de riesgo en personas con antecedentes de fiebre en la provincia de Manu, Madre de Dios, Perú. Rev Peru Med Exp Salud Publica. 2003:20:180-5.

37. Trevejo RT, Rigau-Pérez JG, Ashford DA, McClure EM, JarquinGonzález C, Amador JJ, et al. Epidemic leptospirosis associated with pulmonary hemorrhage-Nicaragua, 1995. J Infect Dis. 1998:178:1457-63

38. Lilenbaum W. Martins G. Leptospirosis in cattle: A challenging scenario for the understanding of the epidemiology. Transbound Emerg Dis. 2014:61:63-8.

39. Garcia JL, Navarro IT. Avaliação sorológica da leptospirose e brucelose em pacientes moradores da área rural do município de Guaraci, Paraná, Brasil. Rev Soc Bras Med Trop. 2001;34(3):301-2.

40. Ramos-Vieira NM, Osório-Cisalpino E, Cota-Koury M. Survey of anti-Leptospira agglutinins in workers from the city of Londrina-Paraná, Brazil. Rev Latinoam Microbiol. 1993:35(2):153-7.

41. Gonçalves DD, Teles PS, Reis CR, Lopes FMR, Freire RL, Navarro IT, et al. Seroepidemiology and occupational and environmental variables for leptospirosis, brucellosis and toxoplasmosis in slaughterhouse workers in the Paraná State, Brazil. Rev. Soc. Bras. Med. Trop. 2006:48(3):135-40

42. Vasconcelos LM, Cisalpino EO, Vieira MNR, Koury MC. Pesquisa de aglutininas anti-Leptospira em diferentes grupos profissionais na cidade de Londrina, Paraná. Ver. Soc. Bras. Med. Trop. 1992:25(4):251-5.

43. Schneider M, Velasco-Hernandez J, Min K-D, Leonel DG, BacaCarrasco D, Gompper ME, et al. The use of chemoprophylaxis after floods to reduce the occurrence and impact of leptospirosis outbreaks. Int J Environ Res Public Health. 2017:14(6):594. 